\title{
Measurement of acoustic reflection characteristics of the human cheek
}

\author{
Naohisa Kamiyama, Nobuhiro Miki, and Nobuo Nagai \\ Research Institute of Applied Electricity, Hokkaido University, \\ N12, W6, Sapporo, 060 Japan
}

(Received 10 July 1989)

\begin{abstract}
The vocal tract wall impedance is considered to be related to the transmission loss in the vocal tract and to the radiation from the wall of the vocal tract, and is important in constructing an acoustic model or a circuit model of the speech production system. The wall impedance has been measured by mechanical methods before. Since the vibration of the wall is considered to be caused by the sound pressure in the vocal tract, we propose an approach for direct measurement of the reflection characteristics of the human cheek from the sound pressure distribution in a uniform tube whose end is fixed on the cheek surface. We obtained similar reflection characteristics between male cheeks and female ones. Quite similar characteristics are obtained for the inner wall of the cheek and the outer. Moreover, the characteristics can be expressed by two acoustic impedance constants. Comparing our impedance parameters with those reported by some other researchers, ours are relatively smaller. We also show the effects of our impedance parameters on the transfer characteristics of the vocal tract.
\end{abstract}

Keywords: Speech production, Impedance, Reflection coefficient, Measurement, Vocal tract

PACS number: 43. 85. Bh, 43. 70. Bk

\section{INTRODUCTION}

For speech synthesis by a vocal tract analogue model, the vocal tract has been modeled as cascaded uniform tubes. The characteristics of the model are determined from its area function and the method of loss evaluation. Two types of loss factors may be considered in the vocal tract. One is originated by the heat conductivity and the viscosity of air, and the other is originated by the vibration of the non-rigid wall of the vocal tract. The flexible vocal tract wall has a finite value of impedance, and the impedance is considered to be related to the transmission loss in the vocal tract and the radiation from the wall of the vocal tract. Thus the measurement of the acoustic impedance is important in the construction of an acoustic model and a circuit model for the speech production system.

Some of the characteristics of the vocal tract wall impedance have been reported by using some analysis methods or direct measurement. Table 1 shows some of the reported values of the vocal tract wall impedance, for reference. J. Suzuki ${ }^{1)}$ compared the formant frequency of the signal of real speech with the formant frequency calculated by the equivalent circuit of the vocal tract, and he estimated the wall impedance which establishes a correspondence between the two formant frequencies. Ishizaka, French and Flanagan ${ }^{2)}$ and $\mathrm{H}$. Suzuki ${ }^{3)}$ evaluated the impedance of the human cheek directly with an accelerator placed in contact with the cheek. In that case, these measured impedances are mechanical impedances.

Since the vibration of the vocal tract wall is considered to be caused by the sound pressure in the vocal tract, we now propose an approach for direct measurement of the sound pressure in a uniform tube whose end is fixed on the cheek; and we 
Table 1 Previously reported values of the vocal tract wall impedance per unit area. $Z_{\mathrm{w}}=B+j(\omega M-K / \omega)$.

\begin{tabular}{lrcc}
\hline & $B^{*}$ & $M^{*}$ & $K^{*}$ \\
\hline Flanagan (1965) & 6,500 & 0.4 & - \\
\hline $\begin{array}{l}\text { Ishizaka, French and } \\
\text { Flanagan (1975) }\end{array}$ & 800 & 2.1 & 84,500 \\
\hline J. Suzuki (1977) & 1,400 & 1.6 & - \\
\hline Lunde (1985) & 650 & 1.38 & - \\
\hline $\begin{array}{l}\text { H. Suzuki and } \\
\text { T. Nakai (1987) }\end{array}$ & 1,600 & 2.12 & 154,900 \\
& 1,150 & 1.96 & 127,200 \\
& 1,170 & 1.87 & 142,800 \\
\hline Measured $(f<325 \mathrm{~Hz})$ & 700 & 0.5 & - \\
$(f \geq 325 \mathrm{~Hz})$ & 1,900 & 0.3 & - \\
\hline
\end{tabular}

* Unit of $B, M$ and $K:\left(\mathrm{g} / \mathrm{s} \mathrm{cm}^{2}\right),\left(\mathrm{g} / \mathrm{cm}^{2}\right)$ and $\left(\mathrm{dyn} / \mathrm{cm}^{3}\right)$

evaluate the acoustic reflection characteristics of the human cheek. The amplitude of the reflection characteristics we obtained is smaller than the characteristics evaluated from the impedance by other researchers. The characteristics of our experimental results can be modeled with two linear impedance circuits. We compute the transfer characteristics of the vocal tract in order to evaluate the effect of our impedance values. Typical change in the first formant can be seen with respect to its frequency and bandwidth for Japanese vowels.

\section{MEASUREMENT TECHNIQUE}

We can obtain the acoustic impedance of a subjected connected at one end of the tube by measuring the distribution of the standing wave in the tube. In this paper, we used two types of measurement methods. In the frequency region above $1 \mathrm{kHz}$, we employ the standing wave ratio (SWR) method. In this region, the loss of the air is negligibly small because the distance between the first node and antinode of sound pressure is short, and the first node is near to the measurement object (the cheek). In the frequency region below $1 \mathrm{kHz}$, however, the distance between the node and antinode of sound pressure is relatively long and the node and antinode appear far from the object, or beyond the measurement limit of the tube. Since we cannot neglect the propagation loss in this case, we employ the transfer function method in the lower frequency region.

\subsection{SWR Method $\left.{ }^{4}, 8\right)$}

The acoustic pressure reflection coefficient $S_{\mathrm{p}}$ is described as follows:

$$
S_{\mathrm{p}}=\frac{Z_{1}-Z_{\mathrm{c}}}{Z_{1}+Z_{\mathrm{c}}}
$$

where $Z_{1}$ is the unknown impedance of a subject (cheek) which is fixed to the end of the tube, and $Z_{\mathrm{c}}$ is the characteristic impedance of the tube. The standing wave ratio in the tube is obtained as

$$
R=\frac{1+\left|S_{\mathrm{p}}\right| \exp \left(-2 \alpha_{\mathrm{c}} d_{\max }\right)}{1-\left|S_{\mathrm{p}}\right| \exp \left(-2 \alpha_{\mathrm{c}} d_{\min }\right)} \exp \left(\frac{\alpha_{\mathrm{c}} \lambda}{4}\right)
$$

where $d_{\min }$ in the distance from the end of the tube to the position of a node, $d_{\max }$ is the distance to the position of an antinode $\left(d_{\max }=d_{\min }+\lambda / 4, \lambda\right.$ : wave length), and $\alpha_{\mathrm{c}}$ is the real part of the propagation constant $\gamma_{\mathrm{c}}=\alpha_{\mathrm{c}}+j \beta_{\mathrm{c}}$.

Measuring $R$ and $d_{\min }$, we can calculate the amplitude $\left|S_{\mathrm{p}}\right|$ and phase $\phi$ of $S_{\mathrm{p}}$ as follows:

$$
\begin{gathered}
\left|S_{\mathrm{p}}\right|=\frac{\left(R^{\prime}-1\right) \exp \left(2 \alpha_{\mathrm{c}} d_{\min }\right)}{R^{\prime}+\exp \left(-\alpha_{\mathrm{c}} \lambda / 2\right)} \\
R^{\prime}=R \exp \left(-\alpha_{\mathrm{c}} \lambda / 4\right) \\
\phi=\pi+2 \beta_{\mathrm{c}} d_{\min } .
\end{gathered}
$$

Note that the measured $d_{\min }$ must be smaller than $d_{\max }$ in the above equations.

The impedance of the subject is calculated by the following equation:

$$
\begin{gathered}
Z_{1}=\frac{M-N}{M+N} Z_{\mathrm{c}} \\
M=R^{\prime} \exp \left(2 \alpha_{\mathrm{c}} d_{\min }\right)+\exp \left(-2 \alpha_{\mathrm{c}} d_{\max }\right) \\
N=\left(R^{\prime}-1\right) \exp \left(j 2 \beta_{\mathrm{c}} d_{\min }\right) .
\end{gathered}
$$

\subsection{Transfer Function Method $^{5-7)}$}

A cascade matrix $\boldsymbol{F}$ between the end of the tube $(x=0)$ and the position $x$ is expressed as follows:

$$
\boldsymbol{F}=\left[\begin{array}{cc}
\cosh \left(\gamma_{\mathrm{c}} x\right) & Z_{\mathrm{c}} \sinh \left(\gamma_{\mathrm{c}} x\right) \\
Z_{\mathrm{c}}^{-1} \sinh \left(\gamma_{\mathrm{c}} x\right) & \cosh \left(\gamma_{\mathrm{c}} x\right)
\end{array}\right] .
$$

Assuming the sound pressure at the position $x_{1}$ and $x_{2}$ as $P_{1}$ and $P_{2}$, and we define the pressure transfer function $H_{\mathrm{c}}$ as:

$$
H_{\mathrm{c}}=P_{2} / P_{1} \text {. }
$$

$Z_{1}$ can be obtained from the following equation:

$$
Z_{1}=\frac{H_{\mathrm{c}} \sinh \left(\gamma_{\mathrm{c}} x_{1}\right)-\sinh \left(\gamma_{\mathrm{c}} x_{2}\right)}{H_{\mathrm{c}} \cosh \left(\gamma_{\mathrm{c}} x_{1}\right)-\cosh \left(\gamma_{\mathrm{c}} x_{2}\right)} Z_{\mathrm{c}} .
$$

We can evaluate the reflection coefficient by substituting Eq. (8) into Eq. (1). 


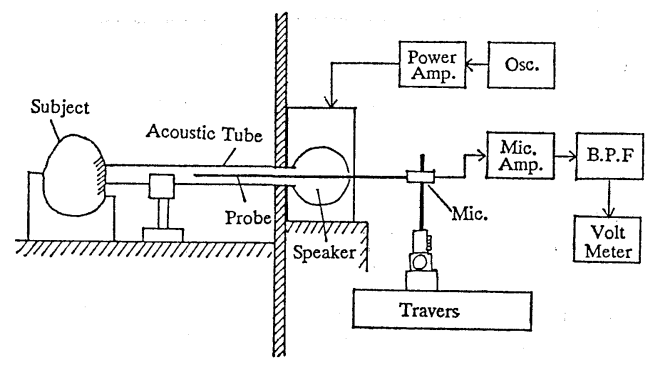

(a)

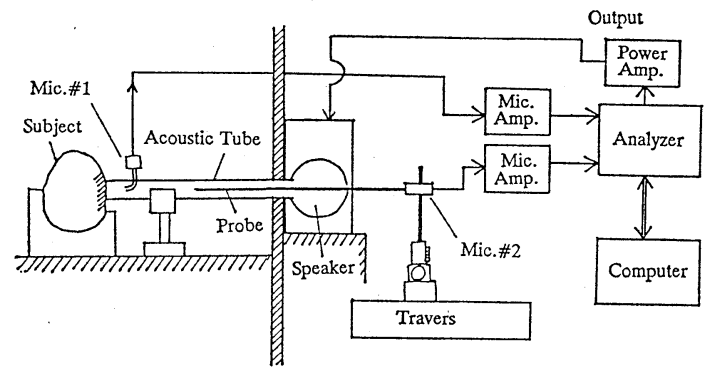

(b)

Fig. 1 Block diagrams of measurement systems. (a) SWR method (b) Transfer function method.

\section{MEASUREMENT EQUIPMENT}

Figure 1 shows block diagrams of the two measurement systems. We fix a subject for measurement to one end of the uniform acoustic tube whose cross section is circular, without any sound leak from the junction between the tube end and the subject. At the other end, pure-tone is fed by the speaker system. And a probe microphone, which is installed on a travers, is employed for measuring the sound pressure in the tube.

The measuring tube is made of acryl resin, $2.3 \mathrm{~cm}$ inside diameter, $3.0 \mathrm{~cm}$ outer diameter, $4.2 \mathrm{~cm}^{2}$ cross-sectional area and $70 \mathrm{~cm}$ length. For the comparison of the influence of the tube area, we also provide another uniform tube whose crosssectional area is $8.2 \mathrm{~cm}^{2}$. The probe microphone is a condenser microphone with a glass probe whose outer diameter and length are $3.0 \mathrm{~mm}$ and $72 \mathrm{~cm}$, respectively. Output signals in the case of the SWR method are amplified by the direct-current amplifier (NEC San-ei 6L06). The signals passing through the bandpass filter (NF E3201) is read with a digital voltmeter. In the case of the transfer function method, we employ a frequency analyzer

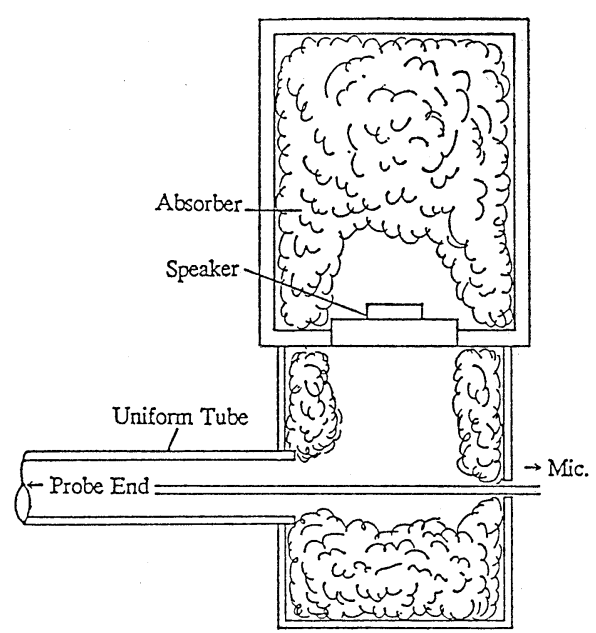

Fig. 2 The part around the sound generator.

(NF S-5720, measurement error $\pm 0.03 \mathrm{~dB}$ and $\pm 0.05 \mathrm{deg}$, typical). Measured amplitudes and phases are stored in the computer by on-line operations. Mic. \#1 is set fixed near the end of probe of Mic. \#2 to receive the reference signals. We measure the sound pressure $P_{\text {mic } 2}$ from Mic. $\# 2, P_{\text {mic } 1}$ from Mic. $\# 1$, and obtain $\boldsymbol{P}_{\text {mic } 2} / \boldsymbol{P}_{\text {mic } 1}$ in complex form. If the reference signals are far from the probe end of Mic. \#2, the phase distribution will be sensitively influenced by a small change of the temperature during the experiment. The accurate position of the probe end of Mic. \#2 can be read by a vernier scale on the travers.

Figure 2 shows the part around the sound generator. In this part, a small hole (its diameter is $1 \mathrm{~cm}$ ) is opened for the probe at the side opposite the uniform tube, and acoustic absorber is stuck to the inner wall. In this system, we can ignore the influence of sound through the side wall of the microphone probe.

The sound speed might vary during the measurement because of the temperature in the tube rising due to the cheek heat. We kept the room temperature at $26 \sim 27^{\circ} \mathrm{C}$ in order to prevent such an influence. 


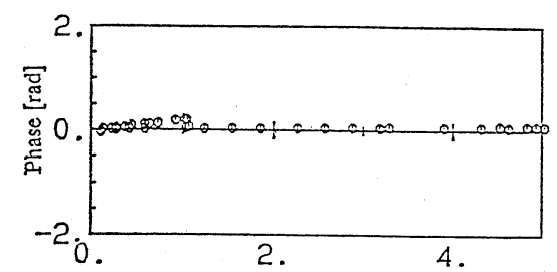

(b)

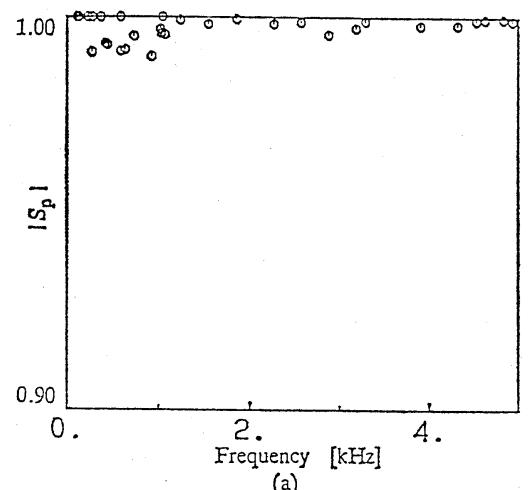

Fig. 3 The reflection characteristics of the acrylic board. (a) amplitude (b) phase.

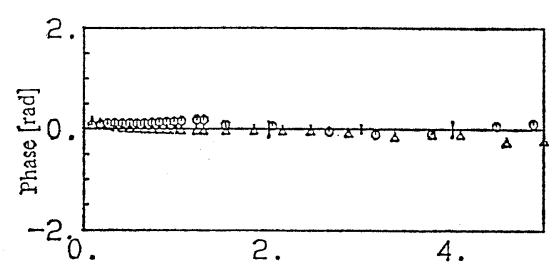

(b)

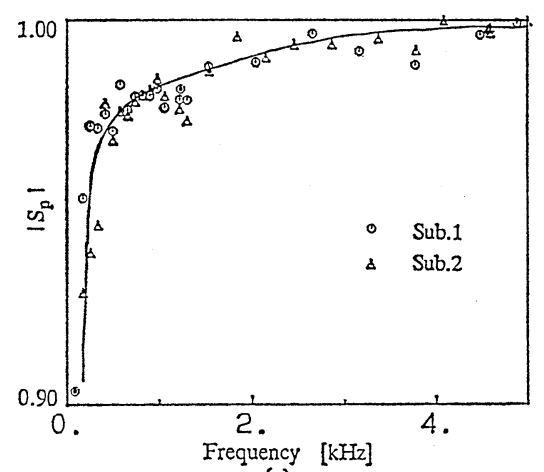

(a)

Fig. 5 The reflection characteristics of human cheeks (Female). (a) amplitude (b) phase.

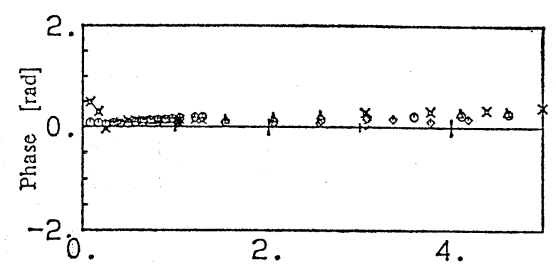

(b)

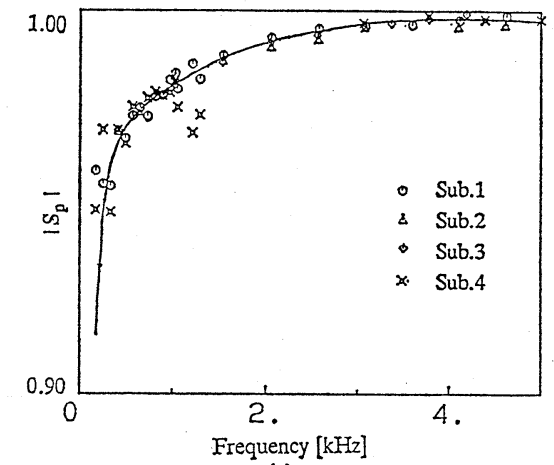

(a)

Fig. 4 The reflection characteristics of human cheeks (Male). (a) amplitude (b) phase.

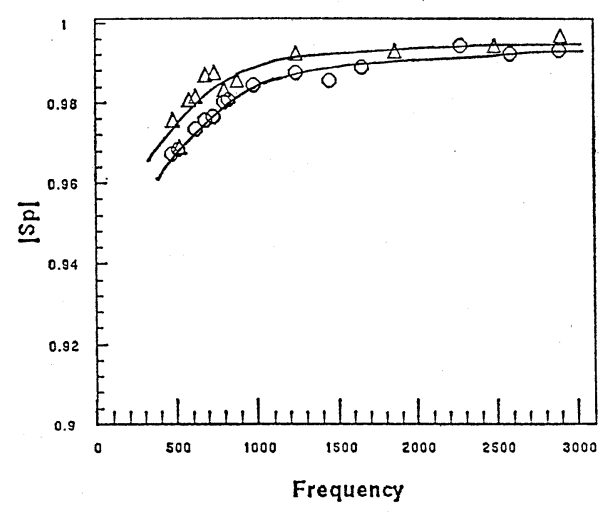

Fig. 6 The amplitude of the reflection characteristics of males' cheeks for two crosssectional area of the acoustic tube. $(O$ : $A=4.2 \mathrm{~cm}^{2}, \triangle: A=8.8 \mathrm{~cm}^{2}$ ). 


\section{N. KAMIYAMA et al.: MEASUREMENT OF REFLECTION CHARACTERISTICS OF CHEEK}

\section{MEASUREMENT AND RESULTS}

\subsection{Preliminary Measurement}

We performed a preliminary measurement on a rigid acrylic board which was fixed on the end of the uniform tube, and evaluated its reflection characteristics. Figure 3 (a) shows the amplitude and (b) the phase of the reflection characteristics of the acrylic board. The transfer function method was employed in the frequency region $85 \mathrm{~Hz} \sim 1.3 \mathrm{kHz}$ and the SWR method in the frequency region $1 \mathrm{kHz} \sim 5 \mathrm{kHz}$ (so also in the following experiments). Values of $\left|S_{\mathrm{p}}\right|$ more than 0.99 were obtained in this measurement. From this result we found that our measurement system was applicable for measurement of the impedance of the cheek.

\subsection{Measurement for the Surface of the Cheek}

Measurement of the acoustic impedance of the cheek was performed for four male and two female subjects with their cheeks fixed on the end of the uniform tube, and with their heads fixed. In the following experiments, the uniform tube of $4.2 \mathrm{~cm}^{2}$ area was used if the area of the tube was not specified explicitly. The sound pressure level in our measurement is about $110 \mathrm{~dB}$ at the antinode of the standing wave. In our measurement, we tried several sound pressure levels from $90 \mathrm{~dB}$ to $110 \mathrm{~dB}$, but the resulting values of the reflection characteristics were almost the same. Figures 4 and 5 show the measurement results of reflection characteristics for males and females, respectively. Solid lines in the (a)s show the approximated curves of the results. The scattering in the data of the females' amplitude seems to be larger than that of the males', mainly in the region of high frequency. However, the general characteristics for the females are quite similar to those of the males, and we cannot find individual differences in the results. The amplitude of the reflection characteristics curves down as frequency decreases. Figure 6 shows the $\left|S_{\mathrm{p}}\right|$ of the males' cheek when the cross-sectional area of the acoustic tube is $4.2 \mathrm{~cm}^{2}$ and when it is $8.2 \mathrm{~cm}^{2}$. In the curves for the measured data in Fig. 6 we find a slight difference, but the two curves are within the range of the scattering in the data for males and females in Figs. 4 and 5. Thus it is considered that influences from a difference in the cross-sectional area of the tube is not large. However, we have not yet ascertained the influence of the cross-sectional area beyond the

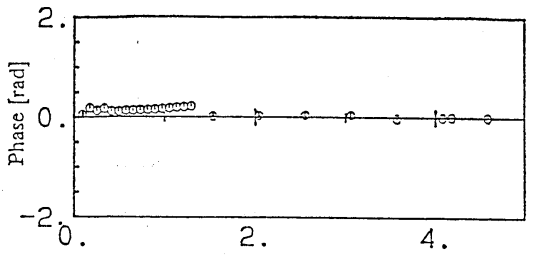

(b)

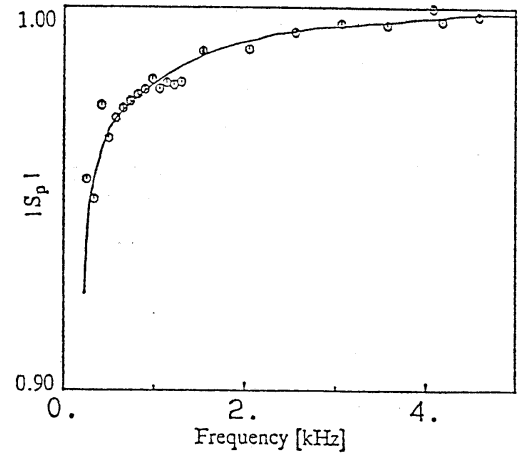

(a)

Fig. 7 The reflection characteristics of the inner wall of the cheek. (a) amplitude (b) phase.

region mentioned

The characteristics of the phase are near zero. However, we can slightly see the linear advance of plotted phase in the ascent of frequency. This is caused by protrusion of the cheek into the measurement tube.

\subsection{Measurement of the Inner Wall of the Cheek}

For the measurement of the inner wall of the cheek, we inserted the measurement tube into the subject's mouth. His mouth is opened enough for the tube end to be fixed on the inner wall of the cheek. Figure 7 shows the experimental results for a male subject who is the subject in Section 4.2. In the amplitude and phase of the reflection characteristics of Fig. 7 we find that these data are similar to those of the outer surface.

\section{COMPARISON OF THE IMPEDANCE VALUES}

As mentioned in Chapter 1, the impedance parameters of the vocal tract wall which have been reported previously are mechanical impedances (per unit area). Now we compute reflection coefficients from these impedances under the assumption that they are acoustic impedance load fixed to the end of 

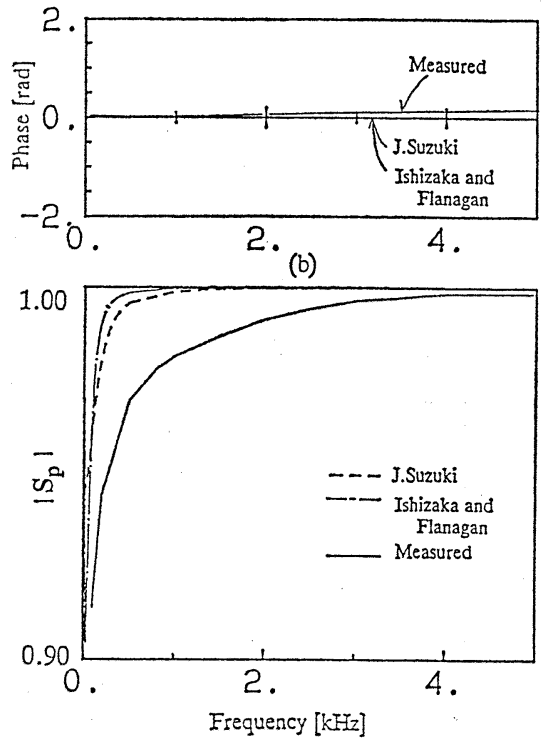

(a)

Fig. 8 Comparison of the reflection characteristics with the previously reported values, (a) amplitude (b) phase.

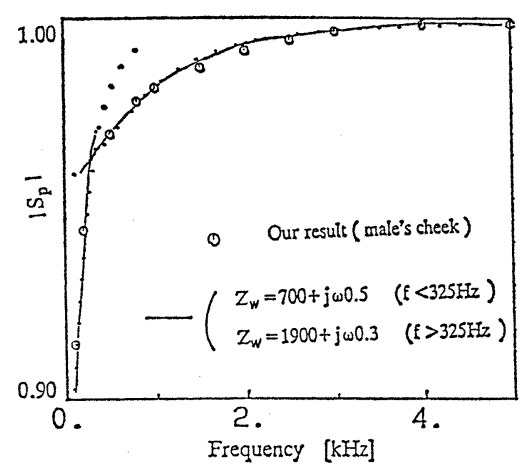

Fig. 9 The reflection characteristics when the acoustic impedance which are adjusted to our results are given.

the uniform tube, and we compare the characteristics of these reflection coefficients with our result. The impedances we used for reference are by Ishizaka and Flanagan in 1975 (the cheek is relaxed by less muscle contraction) and by Jouji Suzuki in 1977. Figure 8 shows the comparison of the three data. Solid lines indicate the approximated curve of our result for the male cheek (Fig. 4). The data presented by Ishizaka and Flanagan and by Suzuki (which we found to be almost the same for all the other values previously reported) show values of $\left|S_{\mathrm{p}}\right|$ of 0.99 in the frequency region above $500 \mathrm{~Hz}$, which means that the wall is almost rigid in this frequency region. Our result shows relatively smaller amplitude than the others.

Besides, we tried to obtain constants for the acoustic impedance (per unit area): $Z_{\mathrm{w}}=B+j \omega M\left(\mathrm{~g} / \mathrm{s} / \mathrm{cm}^{2}\right)$. In the end, we could not find a constant value of impedance which could approximate all the reflection characteristics of our results. We found, however, that the impedance value $Z_{\mathrm{w}}=700+j \omega 0.5$ follows our measured characteristics in the frequency region below $325 \mathrm{~Hz}$, and $Z_{\mathrm{w}}=1900+j \omega 0.3$ follows them in the frequency region above $325 \mathrm{~Hz}$ (shown in Fig. 9). These parameters, especially the imaginary parts, are smaller than the previous values in Table 1. $M$ is a dominant factor to increase $\left|S_{p}\right|$ with respect to the frequency. If we divide the entire frequency region into many narrow sections and search impedances for each section, the better approximation characteristics would be found. The reason we divided it into two sections is to express with fewer parameters, or to avoid complexity.

\section{TRANSFER CHARACTERISTICS}

Figure 10 shows the vocal tract transfer characteristics which are computed from a cascaded uniform tube model of the vocal tract with area functions for Japanese vowels.

The solid line in each figure shows the transfer characteristics when $Z_{\mathrm{w}}=\infty$. The dashed line shows the characteristics when our results are used and the chain line when $Z_{\mathrm{w}}=1400+j \omega 1.6$ (by J. Suzuki, 1977). The first formant frequency is shifted higher by introducing our impedance parameters, and its bandwidth is wider than those from the impedances by other researchers. Note that the question here is not whether the characteristics are similar to real speech signals or not, because the area functions, which show the similar characteristics of the real speech when $Z_{\mathrm{w}}=\infty$, are now used. In this case, relative comparison is the focus. However, the influence looks larger when our parameters are used. One of the reasons of these results is considered that the measured values are under the condition of the vertical incident of the wave, whereas on the contrary, the direction of the propagation wave in the vocal tract is not vertical to the vocal tract wall. 


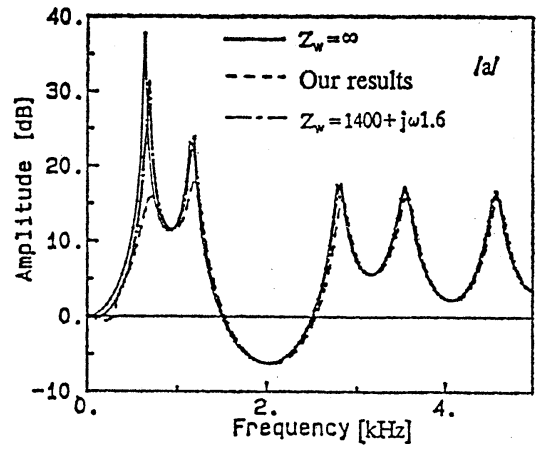

(a)

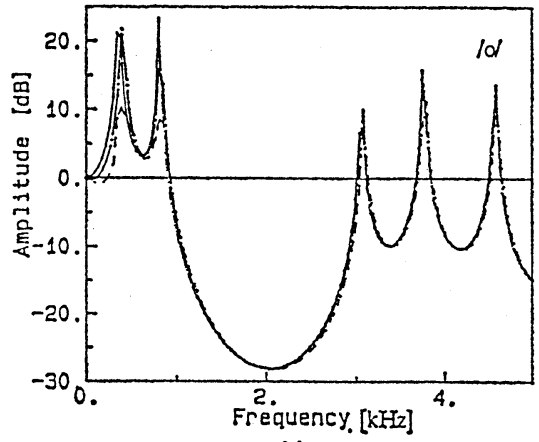

(c)

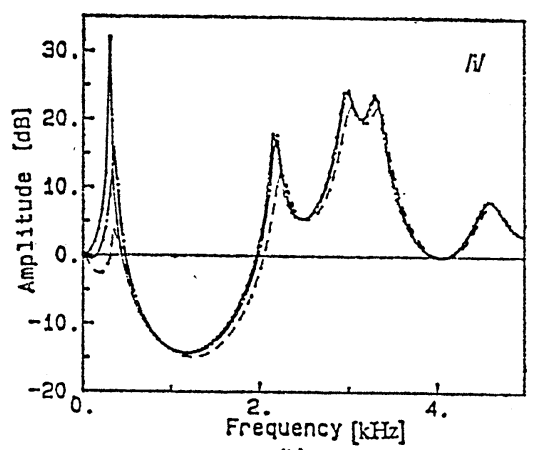

(b)

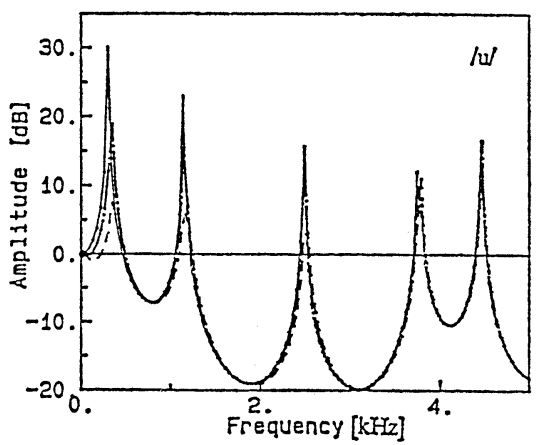

(d)

Fig. 10 Transfer characteristics computed from the cascaded uniform tube model of the vocal tract.

\section{CONCLUSIONS}

We measured the acoustic reflection characteristics of the surface and the inner wall of the human cheek, by the SWR method and the transfer function method. The characteristics have little sexual and individual differences, and are almost the same whether on the surface or on the inner wall of the cheek. The amplitude of the reflection coefficient curves down as frequency decreases and is smaller than the characteristics computed from the impedance parameters which have been reported previously. The characteristics of our measurement can be expressed by two impedance constants which are applied respectively above and below the frequency of $325 \mathrm{~Hz}$. And we investigated influences on the transfer characteristics of the vocal tract when we use our impedance parameters. These influences look somewhat larger, when we directly apply our impedance parameters. It may be required to consider the effects of the incident direction of the wave in order to apply the measured values to the vocal tract model. Moreover the vocal tract also has a wall like the hard palate in addition to the soft cheek. We must investigate further to determine the distribution of the wall impedance in the vocal tract.

\section{ACKNOWLEDGEMENTS}

The authors thank Dr. K. Motoki for his helpful suggestions and support in the experiments, and also the students of our laboratory for their support.

A part of this work was supported by Grant-inAid for Scientific Research on Priority Areas, "Advanced Man-Machine Interface Through Spoken Language," The Ministry of Education, Science and Culture, Japan.

\section{REFERENCES}

1) J. Suzuki, "Discussions on vocal tract wall impedance," J. Acoust. Soc. Jpn. (J) 34, 149-156 (1978) (in Japanese).

2) K. Ishizaka, J. C. French, and J. L. Flanagan, 
"Direct determination of vocal tract wall impedance," IEEE Trans. ASSP-23, 370-373 (1975).

3) H. Suzuki and T. Nakai, "Vocal tract wall impedance and its effect on speech parameters," Res. Rep. Grant-in-Aid for Scientific Research on Priority Areas, PASL 62-11-1 (1987) (in Japanese).

4) "Methods of test for sound absorption of acoustical materials by the tube method," JIS A1405 (1963) (in Japanese).

5) A. F. Seybert and D. F. Ross, "Experimental determination of acoustic properties using a twomicrophone random-excitation technique," J. Acoust. Soc. Am. 61, 1362-1370 (1977).

6) J. Y. Chung and D. A. Blaser, "Transfer function method of measuring in-duct acoustic properties. I. Theory," J. Acoust. Soc. Am. 68, 907-913 (1980).

7) J. Y. Chung and D. A. Blaser, "Transfer function method of measuring in-duct acoustic properties. II. Experiment," J. Acoust. Soc. Am. 68, 914-921 (1980).

8) K. Motoki, N. Miki, and N. Nagai, "A method to measure the area function from distribution of complex sound pressure in the nonuniform acoustic tube," Jpn. IEICE Trans. J72-A, 1222-1229 (1989) (in Japanese).

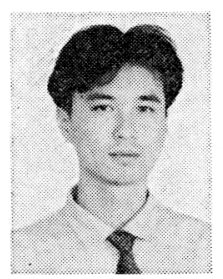

Naohisa Kamiyama was born in Hokkaido, Japan, in 1965. He received the B. Eng. and M. Eng. degrees from Hokkaido University, Sapporo, Japan, in 1988 and 1990 respectively. $\mathrm{He}$ is currently pursuing his studies toward the Dr. Eng. His areas of interest include speech production system and speech signal processing. Naohisa Kamiyama is a studient member of The Acoustical Society of Japan, The IEICE of Japan, and of The Acoustical Society of America.

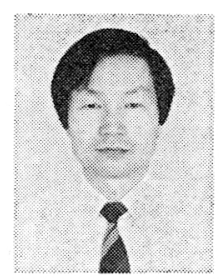

Nobuhiro Miki was born in Kawaguchi, Japan, on October 14, 1945. He received the B. Eng. degree from Tokyo Denki University, Tokyo Japan, 1968, and the M. S. and Ph. D. degrees in electronics from Hokkaido University, Sapporo, Japan, in 1970 and 1974, respectively. From 1974 to 1975 he was a Research Associate at the Simulation Center, Hokkaido University. From 1975 to 1980 he was a Research Associate, and since 1980 he has been an Associate Professor at the Research Institute of Applied Electricity, Hokkaido University. In 1988 he was a Visiting Researcher at KTH, Stockholm, and CID, St. Louis. His current research interests are digital processing of speech, speech production model, and computer simulation of systems. Dr. Miki is a member of the editorial board of The Journal of the Acoustical Society of Japan, a member of the Institute of Electronics, Information and Communication Engineers of Japan, and a member of the IEEE.

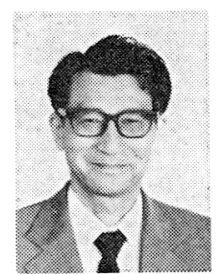

Nobuo Nagai was born in Tokyo, Japan, on January 5, 1938. He received the B. S. and Dr. Eng. degrees from Hokkaido University, Sapporo Japan, in 1961 and 1971, respectively. From 1961 to 1972 , he was a Research Assistant at the Research Institute of Applied Electricity, Hokkaido University. From 1972 to 1980, he was an Associate Professor there, and since 1980 he has been a Professor. He has been engaged in research on distributed-constant networks and digital signal processing. Dr. Nagai is a member of the Institute of Electronics and Communication Engineers of Japan. 